\title{
Transient entrapment neuropathy of the posterior interosseous nerve in violin players
}

\author{
Nicola Maffulli, Francesco Maffulli
}

\begin{abstract}
Eleven white male right handed violin players complained of transient muscular deficit of the extensor compartment of the left forearm during and after prolonged playing. This was associated with paraesthesiae and pain. Relief was achieved keeping the wrist and the elbow flexed, with the supinated forearm held by the contralateral hand. An anatomical study showed changes of the relationship of the posterior interosseous nerve with its surrounding structures with pronation and supination of the forearm. On the basis of the clinical features, the anatomical studies and the response to a simple physiotherapeutic regime, it is suggested that prolonged pronation of the forearm may cause transient entrapment of the nerve.
\end{abstract}

In the past few years musculoskeletal problems in instrumental musicians have been recognised. ${ }^{1}$ Recently, an increasing number of reports have described musculotendinous overuse,$^{23}$ nerve entrapment syndromes ${ }^{45}$ and motor dysfunction ${ }^{67}$ in musicians.

We present the results of the physical examination and treatment of a group of violinists with neuropathy of the posterior interosseous nerve. A necropsy study was undertaken to elucidate the anatomical basis of the syndrome.

\section{Patients and methods}

In the period January 1986-September 1989, eleven white, male, right handed violinists [mean age (SD) $32 \cdot 7(4 \cdot 8)$ years, range $27 \cdot 5-$ $38 \cdot 1$ years] were examined. They had been playing for at least 11 years [mean (SD) 14.3 $(5.8)$ years, range $11-20.5$ years], and were exercising and/or playing at least four hours a day (range four to six hours) for at least six days a week. They complained that halfway through their session they experienced transient muscular deficit of some muscles of the extensor compartment of the forearm, and were unable to perform fine finger movements with the usual speed and precision, due to apparent delay in the muscular contractionrelaxation cycle.

Three patients also complained of muscular pain on the anterior surface of the upper third of the forearm, in four the pain was located close to the lateral epicondyle, whilst in a further four violinists some pain was present on the extensor aspect of the wrist joint. The pain was associated with a burning sensation, and a sensation of sudden muscle bulging. The symptoms were relieved by keeping the elbow and wrist flexed, with the supinated forearm held by the contralateral hand. After three to five minutes in this position, and some frictional massage performed by the player himself, the pain disappeared. This cycle may occur several times in the same session, and the pain mainly occurred in muscles innervated by the posterior interosseous nerve (table 1$){ }^{8}$

No motor nor sensory deficit was detectable by routine physical examination at rest. Cervical spine and peripheral primary muscular and nervous involvement were excluded. The symptoms were reproduced in three patients when the physician pressed with a finger on the anterior aspect of the forearm, close to the supinator muscle, four to five centimetres distal to the elbow joint line. The subject was pronating the forearm and flexing the wrist, keeping the fingers spread in flexion.

From a retrospective analysis of the movements performed while playing, it emerged that the symptoms became more prominent when the forearm was kept in prolonged pronation and the player was beginning to feel fatigued.

Four cadavers were examined, and from these, six upper limbs were dissected. The posterior interosseous nerve was identified and followed along its course. Its relationships with the surrounding structures were studied during pronation and supination movements.

\section{Results}

The posterior interosseous nerve is one of the branches of the radial nerve in the forearm. Originating just distal to the brachioradialis and brachialis muscles, it spirals backwards around the upper end of the radius between the deep and superficial layers of the supinator muscle. The branches for the supinator leave the main nerve in the cubital fossa. In its course, the nerve crosses abductor pollicis longus, reaches the interosseous membrane

\section{Table 1 Muscle groups showing transient deficit}

\section{Left}

$\begin{array}{lr}\text { Extensor digitorum communis } & 11 \\ \text { Extensor carpi ulnaris } & 5\end{array}$
Extensor carpi ulnaris
Extensor carpi radialis brevis 11
5

NB-Some players had muscular deficit in more than one muscle. 
Figure 1 Schematic drawing showing the relationship of the posterior interosseous nerve with the arcade of Frohse during pronation and supination.

Figure 2 Anatomical specimen showing that during pronation movements the nerve comes in contact with the arcade.
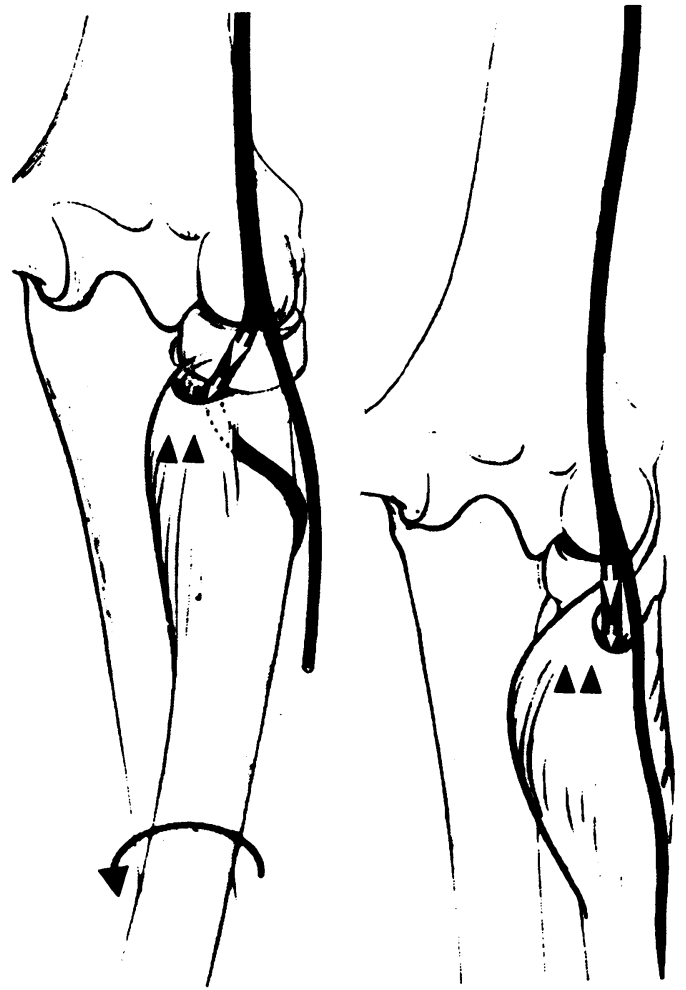

and enters the extensor compartment of the forearm. It supplies the supinator, extensor carpi ulnaris, extensor digitorum communis, extensor digiti minimi, abductor pollicis longus, extensor pollicis longus, extensor pollicis brevis and the extensor indicis. The nerve is also sensory to the interosseous membrane, to the radial and ulnar periosteum, and to the extensor aspect of the wrist and carpal joints. ${ }^{8}$

The supinator muscle consists of two parts,

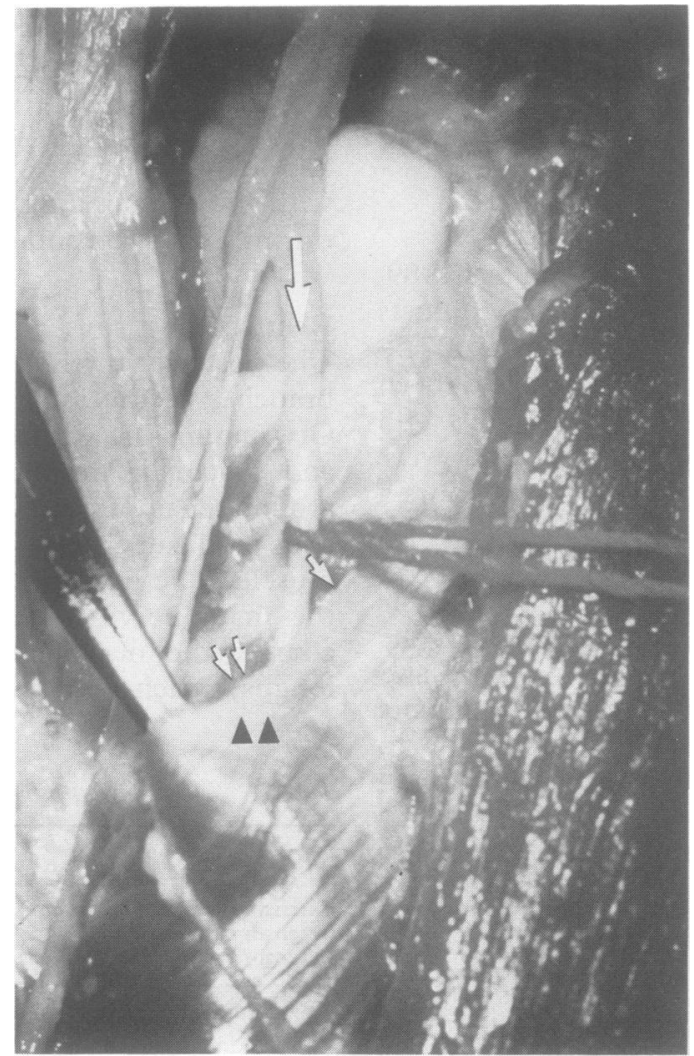

each with its own origin. ${ }^{8}$ The superficial part arises from the tip of the lateral epicondyle, arching downwards for approximately one centimetre. Its fibres attach to the medial aspect of the lateral epicondyle, lateral to the articular surface of the capitulum, thus forming an arch, the arcade of Frohse. ${ }^{9}$ The posterior interosseus nerve passes beneath the arcade. ${ }^{10}$ Normally, the arcade is soft and elastic, and does not compress the posterior interosseus nerve. The relationships of the nerve with the arcade vary according to pronation and supination movements. ${ }^{10}$ When the forearm is supinated, the nerve comes closer to the posterolateral portion of the elbow joint line, and it moves away from it when the forearm is pronated (figs 1-3). When in this position, the major axis of the radial head is just under the posterior interosseous nerve, and compression may occur. The arcade may be membranous or fibrous, ${ }^{10}$ and thus relatively inextensible, as reported by Spinner in $30 \%$ of his series of 50 dissections. ${ }^{10}$ In this case, symptoms may ensue. ${ }^{10}$ In our six anatomical specimens, there was only one case where the lateral part of the arcade was fibrous.

\section{Treatment}

All the patients were advised to abstain for two to three weeks from playing their instrument. During this period, massage, ultrasound therapy and technical coaching were performed. In two subjects, the syndrome recurred a short while after the end of the therapy ( 14 days and 24 days, respectively). In these cases, the arm was put in an above-elbow plastic cast with the elbow flexed at $90^{\circ}$, the forearm supinated

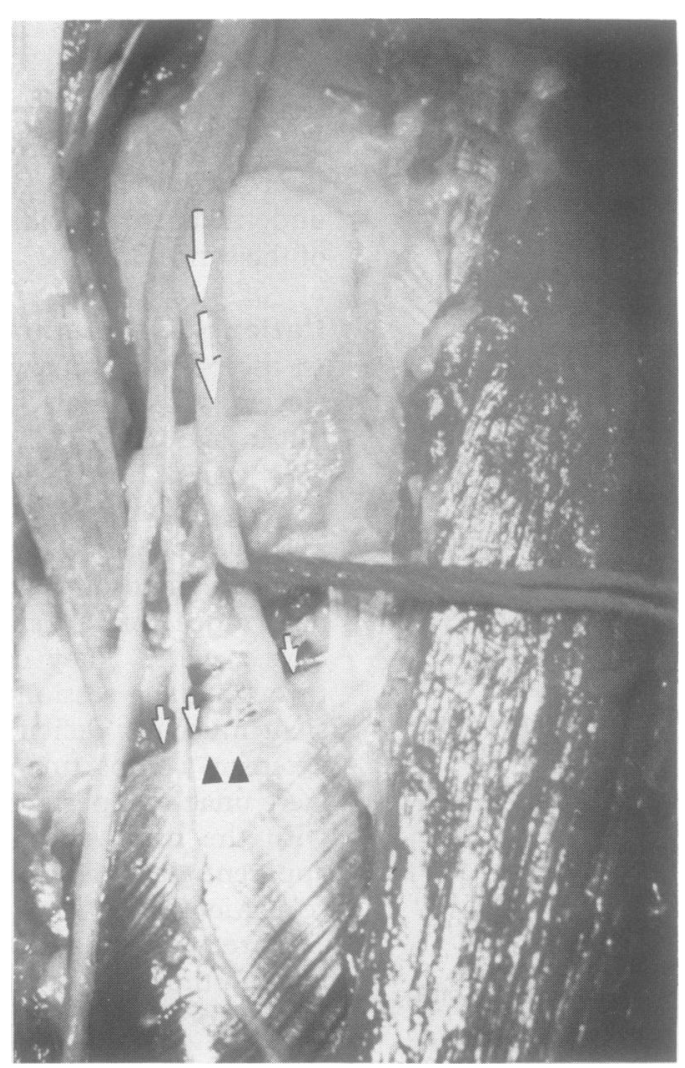

Figure 3 Anatomical specimen showing that during supination movements the nerve is not in contact with the arcade. 
and the wrist in neutral position for ten days. Physiotherapy and technical coaching were continued.

After this further period of treatment, another recurrence occurred. After a final period of conservative treatment, the nerve was explored. The posterior interosseous nerve was swollen, and the arcade of Frohse was fibrotic in the area of contact with the nerve. The nerve was accurately dissected from its neighbouring structures, and the wound sutured in layers. The arm was put in a sling, and early mobilisation suggested. After ten days, gentle physiotherapy was started. The patient has remained fully asymptomatic for the past seven months.

\section{Discussion}

Arm pain, cramps, paraesthesiae and diminished dexterity are quite common among instrumental musicians. ${ }^{5}$ Nerve entrapment syndromes are frequently the cause of such symptoms. ${ }^{5}$ The symptoms described are typical, and once presented with the first case, the others are relatively easy to diagnose. A symptomatic therapeutic approach is not advisable. If the syndrome is not recognised, it may evolve into a permanent paralysis of the posterior interosseous nerve.$^{10}$ In this case, the only solution is surgical exploration. ${ }^{10}{ }^{11} \mathrm{An}$ inextensible fibrous or membranous arcade of Frohse may account for a paralysis, but, as it is relatively rare, ${ }^{11}$ it is unlikely that all the violinists examined have a fibrous arch.

Compression neuropathies are produced by external pressure, and the term entrapment is generally referred to nerve compression at a specific site. The site itself is often predictable from either normal or pathological anatomical and physiological features. The basis of the syndrome in these players is probably functional more than anatomical. Due to repeated contraction and relaxation associated with small changes in pronation and supination with the forearm held predominantly pronated, the posterior interosseus nerve may undergo continuous microtrauma, and pain and functional limitation may ensue. Also, violin playing requires prolonged isometric contraction of the forearm muscles, on which contraction of the fingers flexors are superimposed. Vascular engorgement of the contracted forearm muscles may occur, ${ }^{12}$ with consequent enlargement of the extensible muscle belly, which becomes less elastic than at rest. If this is the case, the posterior interosseus nerve can be compressed by an anatomically normal arcade of Frohse, and the symptoms may ensue. The continuous microtrauma caused by the contact of the nerve with the muscle may induce trauma, inflammation, scarring and formation of fibrous tissue.

Both these hypotheses may account for the characters of the syndrome. The symptoms are transient, and are eased by rest and frictional massage. At rest, the blood accumulated in the muscle flows from it, and the gentle massage helps the flow. A period of rest proves beneficial, as the forearm muscles undergo hypotrophy, and the nerve is not exposed to prolonged microtrauma.

1 Fry $\mathrm{HJH}$. Overuse syndrome in musician. Med J Aust 1987;146:390.

2 Fry HJH. Overuse syndrome in musician. 100 years ago. An historical review. Med J Aust 1986,145:620-5.

3 Lederman RJ, Calabrese LH. Overuse syndrome in instrumentalists. Med Probl Perform Art 1986;1:7-11.

4 Knishkowy $B$, Lederman RJ. Instrumental musicians with upper extremity disorders. A follow up study. Med Probl Perform Art 1986;1:85-99.

5 Lederman RJ. Nerve entrapment syndromes in instrumental musicians. Med Probl Perform Art 1986;1:45-8.

6 Critchley M, Henson RA. Music and the brain. London: Heinemann, 1977.

7 Newmark J, Hochberg FH. Isolated painless manual incoordination in 57 musicians. J Neurol Neurosurg Psychiatry 1987;50:291-5.

8 Last RJ. Anatomy. Regional and applied, 7th ed. Edinburgh: Churchill Livingstone, 1984

9 Frohse F, Frankel M. Die Muskeln de menschlichen Armes. Jena, 1908. (Cited in Spinner M. The arcade of Frohse and its relationship to posterior interosseus nerve paralysis. $J$ Bone Joint Surg 1968;50B:809-12.

10 Spinner $M$. The arcade of Frohse and its relationship to posterior interosseus nerve paralysis. J Bone Joint Surg 1968;50B:809-12.

11 Sharrard WJW. Posterior interosseous neuritis. J Bone Joint Surg 1966;48B:777-80.

12 Astrand P-O, Rodahl K. Textbook of work physiology, 3rd ed. New York: McGraw-Hill, 1986. 